\title{
Vortex-antivortex dynamics driven by spin-torque in a nanocontact
}

\author{
C.E. Zaspel \\ University of Montana-Western, Dillon, MT 59725, USA \\ E-mail: Craig.Zaspel@umwestern.edu \\ V.E. Kireev \\ Institute of Magnetism, NASU, 36-B Vernadskii Ave., Kiev 03142, Ukraine
}

Received March 13, 2015, published online August 25, 2015

\begin{abstract}
A spin-polarized current in a nanocontact has been shown to induce the formation of a magnetic vortex at the nanocontact by the Oersted field, and spin-torque drives the vortex core in an elliptical orbit about the nanocontact. For the case of an external in-plane magnetic field in an extended free layer, the magnetization will be uniform far from the nanocontact, implying that vortex formation must be accompanied by the formation of an antivortex. Using the Thiele approach to describe the vortex-antivortex dynamics it is shown that the frequency of gyrotropic motion of the vortex is a function of the nanocontact current which is linear for large vortex-antivortex separations and it becomes nonlinear as the separation is decreased. The equilibrium vortexantivortex separation can be controlled by the nanocontact current as well as the external magnetic field.

PACS: $75.10 . \mathrm{Hk}$ Classical spin models;

75.75.Jn Dynamics of magnetic nanoparticles;

73.90.+f Other topics in electronic structure and electrical properties of surfaces, interfaces, thin films, and low-dimensional structures.
\end{abstract}

Keywords: magnetic vortex, nanocontact, spin torque.

\section{Introduction}

Highly nonuniform spin distributions with nontrivial topology, such as magnetic vortices, domain walls, as well as other spin structures currently have great perspectives for usage in spintronic and magnonic devices. The LandauLifshitz equation [1], which was first introduced 80 years ago as a phenomenological equation to describe the dynamics of magnetization field, is the best tool for description of such states. The extremely important point is that this equation allows significant simplification of the problem of soliton dynamics through the collective variable approach. For the gyroscopic motion of the magnetic vortex this equation was first derived by Thiele [2]. Presently the Thiele equation is widely used for description of vortex oscillations in magnetic nanoparticles and their ordered arrays, see [3-10] and the article of G.M. Wysin in this Special Issue [11].

Magnetic oscillations in nanocontact systems [12-14] can be generated by spin-torque $[15,16]$ effects where damping is compensated resulting in self-sustained oscilla- tions with gyrotropic motion in the sub-GHz range [3,4] of the vortex as an example having potential applications. The magnetic vortex is a stable structure that can be the actual ground state of a ferromagnetic nanodisk owing to the confining nature of a disk; in addition, the vortex has topological charge, enhancing the stability of this structure. Recently gyrotropic oscillations driven by spin-torque have been observed [17-19] in nanopillar structures. In a large nonconfined ferromagnetic film the ground state is the uniform ferromagnetic state, but the Oersted field about a nanocontact current can induce vortex formation [20-24] with spin-torque driving gyrotropic motion. However, if the magnetization far from the nanocontact remains uniform this implies the formation of an antivortex if topological charge is to be conserved. In this article the motion of a vortex formed at a nanocontact driven by spin torque is analytically investigated taking into account topological charge conservation and the interaction with the antivortex.

Some of the earliest [20] experimental work on the dynamics of nanocontact systems was done with an in-plane external field and the time-dependent magnetization driven 
by a spin-polarized current. A simplified picture of the nanocontact is shown in Fig. 1 where the fixed-layer magnetization as well as the external field are in-plane and the layers extend much further than the nanocontact. In these systems the magnetization dynamics were investigated through the giant magnetoresistance (GMR) effect where the voltage variation across the nanocontact is a measure of the time-dependent magnetization at the nanocontact. For small field values (up to $4 \mathrm{mT}$ ) a high amplitude sub-GHz mode was observed as well as additional lower amplitude higher harmonics. These modes also exhibit hysteresis effects where a critical current of about $10 \mathrm{~mA}$ is required for the formation of the sub-GHz mode, but the mode persists as the current is decreased to about $4 \mathrm{~mA}$ with a weak dependence of frequency on current. Later numerical and analytical investigations [21] have indicated that the vortex forms in the Oersted potential well and spin-torque forces the vortex core out of the nanocontact into an elliptical orbit about the nanocontact center. Analytical results in particular have shown that the orbital frequency is a linear function of the current and the orbital radius only depends on the device parameters; however, this was done for the case of the single rigid vortex. In the numerical simulations [21] initial vortex-antivortex (VA) formation was observed with the antivortex forced outside of the system possibly due to the limiting system size, and in most previous analytical work the antivortex was ignored because of complications to the Landau-Lifshitz equation. Clearly, the orbital motion of the vortex about the nanocontact will result in a timedependent magnetization at the nanocontact and a corresponding oscillating voltage owing to the GMR effect, but the effect of an antivortex on the observed GMR voltage is not understood. Indeed, for the case of the wellseparated VA pair the antivortex will have negligible effect on the GMR voltage. In order for the antivortex to have an observable effect it is necessary to have a large enough VA interaction, and it will be shown that the interaction depends on both the nanocontact current as well as the magnitude of an in-plane magnetic field.

Possible technological applications involving the dynamics of magnetic vortices are microwave oscillators [18]

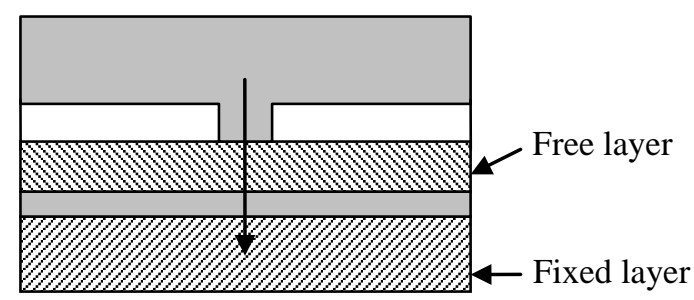

Fig. 1. Nanocontact setup with gray representing nonmagnetic conductors, crosshatch represents magnetic layers and white represents an insulator. The current is along the nanocontact axis and an external field as well as the magnetization are in the layers perpendicular to the axis. constructed from nanopillars or nanocontacts driven by spin torque. The frequencies of these oscillators can be controlled by a relatively low current density, the emission has a narrow linewidth, and they can operate without an external magnetic field. It has further been shown that the oscillation frequency is stable and can be adjusted [25] through the current with a very short (nanosecond) relaxation time. A recent advance has been the synchronization $[23,26,27]$ of these oscillators, and the importance of the antivortex is enhanced by the recent development of arrays of nanocontact oscillators [23,28,29] that are synchronized through intermediate antivortices. The motivation for this research is a better understanding of the effect of the antivortex on vortex dynamics in the single nanocontact system when the VA interaction is significant.

\section{Vortex-antivortex dynamics}

To investigate vortex dynamics through the GMR effect we include the complete vortex-antivortex pair having zero $\pi_{1}$-topological charge by introduction of a small external magnetic field. This in-plane field will fix the magnetization direction far from the nanocontact and a large enough Oersted field then results in a VA pair, stable against small perturbations. For the static VA configuration as illustrated in Fig. 2, the vortex is at the nanocontact center defined to be the origin, the antivortex is on the $y$-axis, and the external field defines the $x$-direction. The coordinate system used to describe VA positions if shown in Fig. 3, where the two origins are at the static equilibrium positions of the vortex and antivortex. The vortex is in a potential well at the nanocontact resulting mainly from the current, and as in previous models the restoring force results in sub-GHz

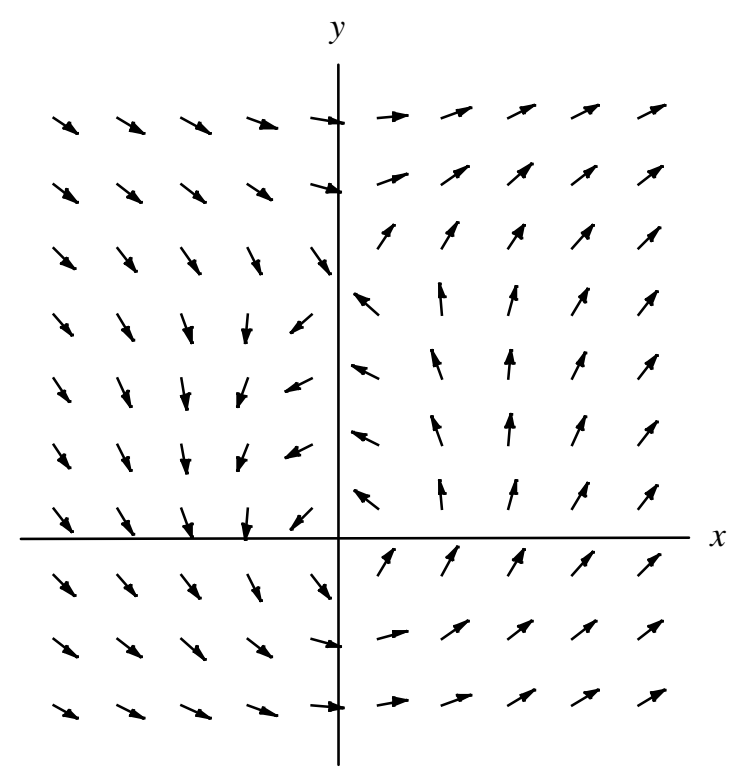

Fig. 2. The vortex-antivortex structure with the vortex centered on the nanocontact. The external field is in the $x$-direction and the antivortex is at an energy minimum on the $y$-axis. 


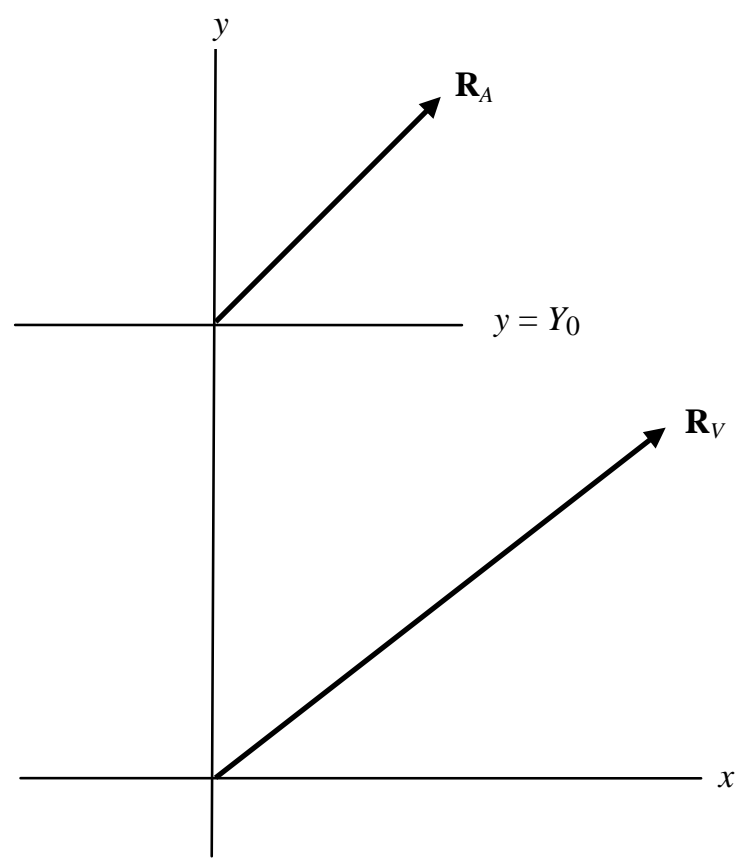

Fig. 3. Coordinate system for the individual vortex and antivortex, where the vortex system is centered at the nanocontact (origin of the $x, y$ system) and the antivortex is centered on the $y$-axis.

vortex motion about the nanocontact center. The antivortex, on the other hand, is in a potential well defined by both the nanocontact current and Zeeman field. Therefore, vortex and antivortex motion is determined by these two conservative potentials as well as spin-torque and dissipation.

To describe the dynamics we begin with the LandauLifshitz equation with Gilbert dissipative term and including spin-torque, $\Gamma_{S T}$

$$
\frac{\partial \mathbf{m}}{\partial t}=\frac{\gamma}{\mu_{0} M_{S}} \frac{\delta W}{\delta \mathbf{m}}+\alpha \mathbf{m} \times \frac{d \mathbf{m}}{d t}+\Gamma_{S T} .
$$

Here $\mathbf{m}=(\sin \theta \cos \phi, \sin \theta \sin \phi, \cos \theta) / M_{s}$ is the normalized free-layer magnetization in terms of polar and azimuthal angles, $M_{s}$ is the saturation magnetization, and $\alpha$ is the Gilbert damping parameter. The torque for a nanocontact current, $I$ and fixed layer polarization, $\hat{p}$ is given by

$$
\Gamma_{S T}=-\sigma I[\mathbf{m} \times(\hat{p} \times \hat{m})],
$$

where the constant, $\sigma=\hbar \gamma P$ / $\left(e \mu_{0} M_{s} 4 \pi r_{0}^{2} L\right)$ is determined by nanocontact parameters such as the nanocontact radius, $r_{0}$, the free-layer thickness, $L$ and $P \leq 1$ is a polarization efficiency.

In the following the collective coordinates for the positions of the vortex and antivortex cores, $\mathbf{R}_{V, A}$ (as defined in Fig. 3) will be used as dependent variables rather than the normalized magnetization. This is accomplished through conversion of Eq. (1) to a Thiele form [2] to give a non-
Newtonian equation of motion including conservative and nonconservative forces on the vortex and antivortex,

$$
\mathbf{G} \times \frac{d \mathbf{R}_{V, A}}{d t}+\frac{\partial W}{\partial \mathbf{R}_{V, A}}=-\alpha D \frac{d \mathbf{R}_{V, A}}{d t}+\mathbf{F}_{S T},
$$

where $\mathbf{G}=2 \pi \mu_{0} M_{s} q \hat{z} / \gamma$ is the gyrovector for a vortex having core polarization, $q= \pm 1$, and $W$ is the energy from which the conservative forces are derived. The first nonconservative force on the right hand side is the Gilbert damping force with

$$
D=\frac{\mu_{0} M_{s}}{\gamma} \int(\nabla \mathbf{m})^{2} d^{2} r
$$

The spin-polarized current force is obtained from $\Gamma_{S T}$ in terms of an integral involving the magnetization polar and azimuthal angles,

$$
F_{S T}=-\sigma I \frac{G}{2 \pi} \int_{N C} \sin ^{2} \theta \nabla \phi d^{2} r,
$$

where the integration is over the nanocontact area and $I$ is the nanocontact current. Note that despite the fact that the general formula for the force contains $q= \pm 1$, the sign of the gyroconstant for the vortex and antivortex is the same, because their winding numbers are opposite.

The conservative force on the left hand side of Eq. (3) is obtained from the energy, $W=W_{\text {ex }}+W_{M S}+W_{O e}+W_{Z}$ including the sum of exchange, magnetostatic, and Zeeman terms. There are two Zeeman contributions to the energy with $W_{O e}$ representing the energy from the Oersted-Ampere field (referred to as the Oersted energy), and $W_{Z}$ is the energy from a uniform external field (referred to as the Zeeman energy). To evaluate each of these terms the vortexantivortex ansatz

$$
\phi(r, \chi)=\tan ^{-1}\left(\frac{x-X_{V}}{y-Y_{V}}\right)-\tan ^{-1}\left(\frac{x-X_{A}}{y-Y_{0}-Y_{A}}\right),
$$

is used, where $\left(X_{V, A}, Y_{V, A}\right)$ are the positions of the vortex and antivortex cores relative to their respective static equilibrium positions in Fig. 3 and $Y_{0}$ is the static equilibrium position of the antivortex on the $y$-axis. This is part of the well-known Belavin-Polyakov soliton [30] in the isotropic ferromagnet resulting the structure shown in Fig. 2 with the magnetization in the $\hat{x}$-direction far from the vortexantivortex pair. Note that the complete solution also includes an expression for the polar angle, but owing to magnetostatic effects in the ferromagnetic film it is assumed that the polar angle, $\theta(r, \chi)=\pi / 2$ except at the vortex and antivortex cores where $\theta \rightarrow 0$ within a very small area of radius the order of the exchange length, $l_{0}$. Also because of the symmetry of this ansatz the integral giving the magnetostatic energy from the magnetostatic volume charge $(\nabla \cdot \mathbf{m})$ is zero, and there is a small contribution from effective surface magnetostatic charge at the vortex 
core. Moreover, the exchange energy has a log-dependence on the VA separation resulting in a negligible force on the vortex or antivortex for typical VA separations. Thus, the Zeeman and Oersted energies give the dominant contributions to the conservative forces in the Thiele equation.

\section{Vortex-antivortex energy}

Next, contributions to the Oersted and Zeeman energies are calculated. Referring to the nanocontact current structure of Fig. 1, the Oersted field is approximated by the field produced by an infinite cylindrical conductor of radius $r_{0}$, with $B=\mu_{0} I r / 2 \pi r_{0}^{2}$ inside the cylinder and $B=\mu_{0} I / 2 \pi r$ outside of the cylinder.

For calculation of the energy in the symmetry of the Oersted field it is convenient to express the vortex and antivortex positions in polar coordinates centered at their respective static equilibrium positions $\left(R_{V, A} \cos \varphi_{V, A}, R_{V, A} \sin \varphi_{V, A}\right)$ where the vortex polar coordinates are centered at the origin and the antivortex polar coordinates are centered at $\left(r=Y_{0}, \chi=\pi / 2\right)$ as shown in Fig. 3 . Then the energy is expressed as

$$
W=-\frac{\mu_{0} M_{s} I}{2 \pi} a-M_{s} B b,
$$

where

$$
a=\int_{l_{0}}^{0} \int_{0}^{2 \pi} m_{\chi} \frac{r^{2}}{r_{0}^{2}} d r+\int_{r_{0}}^{R} \int_{0}^{2 \pi} m_{\chi} d r, b=\int_{l_{0}}^{R} \int_{0}^{2 \pi} \cos \phi r d r d \chi
$$

and the integration is over a polar $(r, \chi)$ coordinate system with its origin at the nanocontact center. Here $m_{\chi}=\sin \phi \cos \chi-\cos \phi \sin \chi$ is the tangential component of the normalized magnetization and $R$ is an upper limit of the radial integration to be determined later.

At this point it is convenient to write the dependence of the energy on deviations of the vortex and antivortex from their equilibrium positions, with $m_{\chi}$ and $\cos \phi$ expanded in series of $R_{V}$ and $R_{A}$ referring to the coordinates defined in Fig. 3. This results in series of the form,

$$
\begin{aligned}
& m_{\chi}=a_{0}+a_{1} R_{V}+a_{2} R_{A}+a_{11} R_{V}^{2}+a_{22} R_{A}^{2}+a_{12} R_{V} R_{A}+\cdots \\
& \cos \phi=b_{0}+b_{1} R_{V}+b_{2} R_{A}+b_{11} R_{V}^{2}+b_{22} R_{A}^{2}+b_{12} R_{V} R_{A}+\cdots,
\end{aligned}
$$

where the coefficients are integrals over $(r, \chi)$ which are functions of $Y_{0}, I$, and $B$ as well as $\phi_{A}$ and $\phi_{V}$. The forms of these coefficients are presented in Appendix A. For evaluation of these coefficients it is first necessary to obtain values for $Y_{0}$ as a function of both current and external field. This can be done by minimization of the energy using the lowest order terms, $a_{0}$ and $b_{0}$ with the vortex fixed at the nanocontact center and the antivortex on the $y$-axis. However, it is remarked that this minimization shows that the antivortex tends to form where the Zeeman and Oersted fields cancel on the $y$-axis, the position of which is
$Y_{0}=\mu_{0} I / 2 \pi B$. This simple result is accurate to $1 \mathrm{~nm}$ when compared with the more accurate numerical minimization. Moreover, as expected, the linear terms evaluated at the vortex and antivortex equilibrium positions will not contribute to the energy since this is indeed an energy minimum.

Next the upper limit is estimated by considering how an external magnetic field has an effect on the value of $R$. Notice that the ansatz of Eq. (6) only gives a magnetization along the field ( $\hat{x}$-direction) as $R \rightarrow \infty$. However, in a nonzero field it is expected that the magnetization will align with the field at a finite value of $R$, so the integration limit is defined to be where this alignment occurs when the external field is taken into consideration. This value is determined by treating the Zeeman energy as a perturbation from which the change in azimuthal angle relative to the ansatz, $\phi^{\prime}$ is obtained. Next Eq. (6) is approximated for $r>>Y_{0}$ to find an approximate small angle for the ansatz, $\Delta \phi$. Finally the condition, $\Delta \phi+\phi^{\prime}=0$ is used to estimate $R$. The details of this upper-limit determination are in Appendix B, and Fig. 4 shows the linear dependence of $R$ on the nanocontact current for fields of $1 \mathrm{mT}$ and $3 \mathrm{mT}$.

All terms in Eqs. (8), (9) that include the antivortex coordinate will converge according to the small parameter, $R_{A, V} / Y_{0}$ but the terms in the series including only the vortex coordinate, $R_{V}$ converge too slowly for expression as a series; therefore, it is necessary to numerically evaluate the energy as a function of $R_{V}$ with $R_{A}=0$. These results are shown is Fig. 5 for a nanocontact current of $12 \mathrm{~mA}$ in an external field of $3 \mathrm{mT}$ with a nanocontact radius of $50 \mathrm{~nm}$. Notice that this energy is approximately quadratic inside the nanocontact and it is a linear function of $R_{V}$ outside of the nanocontact as was established [21] previously. For all other terms the expansions of $m_{\chi}$ and $\cos \phi$ converge and they are useful to obtain analytical results regarding the asymmetry ( $\phi_{V}$ and $\phi_{A}$ dependence) of the VA interaction. Using the forms of the coefficients in Appendix $\mathrm{A}$ and numerical evaluation of the integrals yields the general form of the energy to second order in displacements,

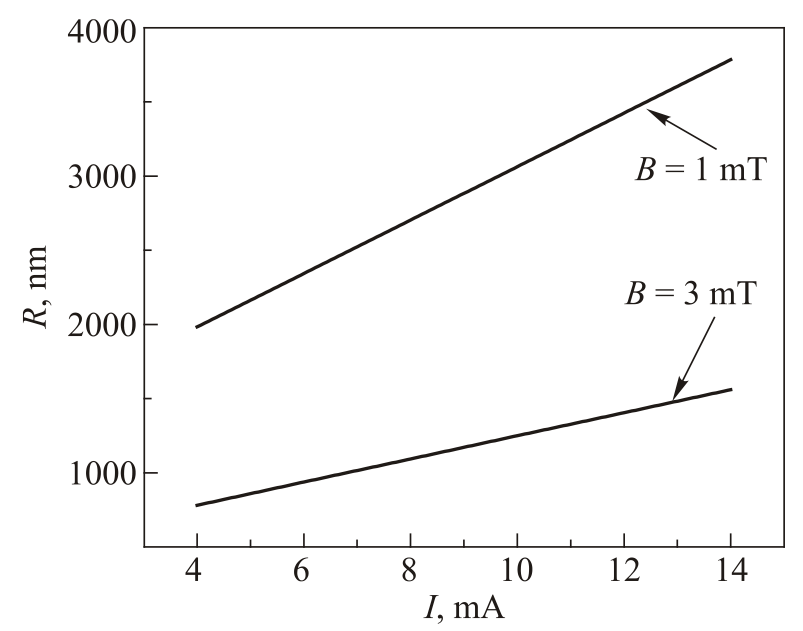

Fig. 4. The upper limit, $R$ versus current for external fields of $1 \mathrm{mT}$ and $3 \mathrm{mT}$. 


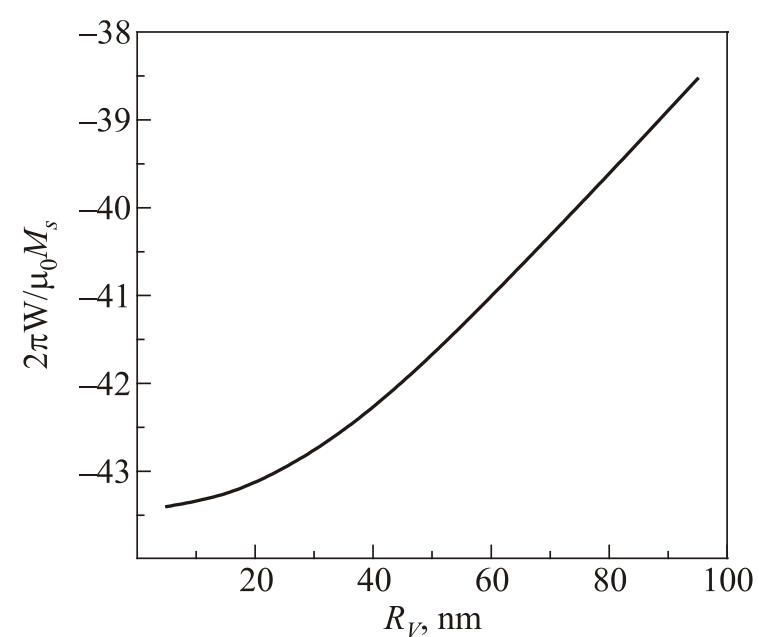

Fig. 5. The energy versus vortex displacement, $R_{V}$ with a current of $12 \mathrm{~mA}$ and an external field of $3 \mathrm{mT}$.

$$
\begin{gathered}
W=\frac{\mu_{0} M_{s}}{2 \pi}\left[k_{1} R_{V}+\frac{1}{2} k_{2} R_{A}^{2}+k_{13} R_{V} R_{A} \cos \phi_{V} \cos \phi_{A}+\right. \\
\left.+k_{12} R_{V} R_{A} \sin \phi_{V} \sin \phi_{A}\right]
\end{gathered}
$$

where the main contribution to anisotropy is from the VA interaction. The coefficient $k_{1}$ is obtained from the slope of the linear region of Fig. 5 and this coefficient also exhibits the most significant current variation with a slight dependence on the external field. The dependence of $k_{1}$ on current for fields of $1 \mathrm{mT}$ and $3 \mathrm{mT}$ is shown in Fig. 6 where a very weak dependence on $B$ is noticed. All of the quadratic parameters in Eq. (10) are calculated by integration of the coefficients in the series of Eqs. (8), (9) given in Appendix A. Notice that these coefficients have a weak dependence on current compared to $k_{1}$ as noticed in Fig. 7. The only significant dependence occurs for the case of small current $(I<8 \mathrm{~mA})$ and large field $(B=3 \mathrm{mT})$ when the VA separation is smallest.

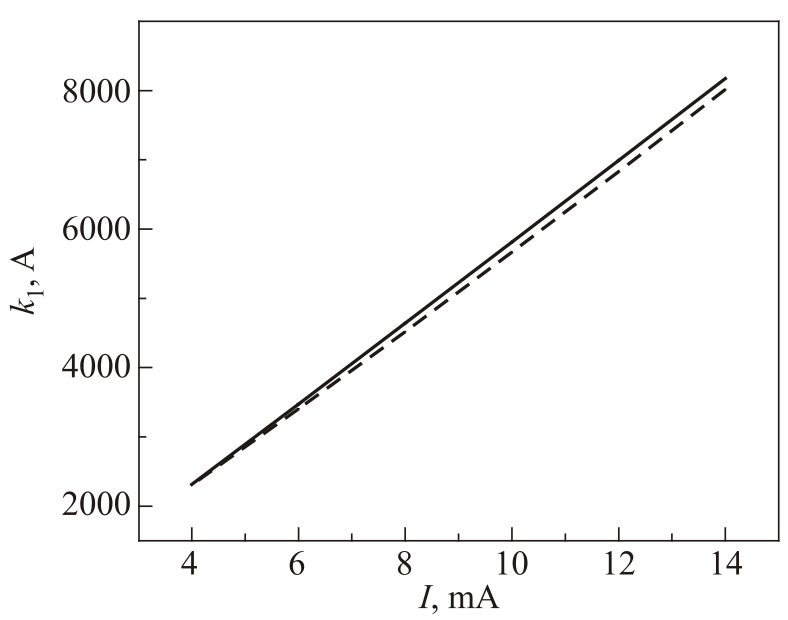

Fig. 6. The parameter $k_{1}$ versus current, $I$ for external fields of $1 \mathrm{mT}$ (dashed) and $3 \mathrm{mT}$ (solid).

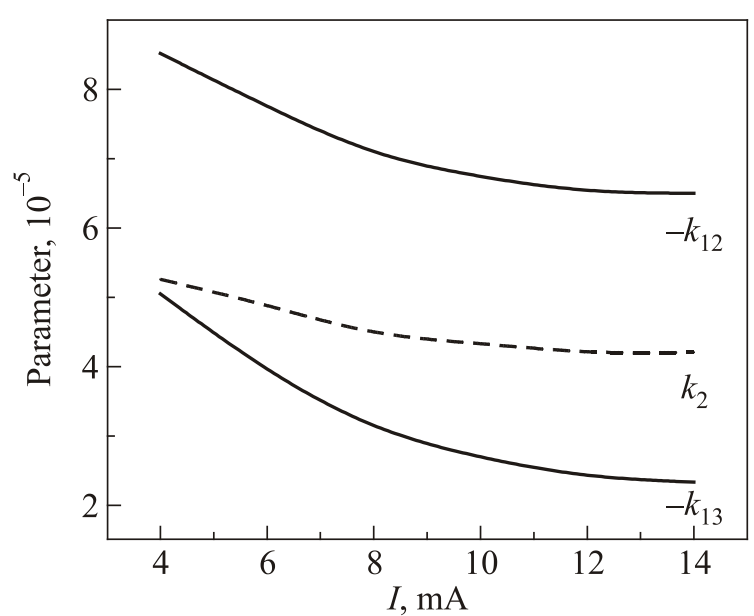

Fig. 7. The antivortex VA interaction parameters, $k_{2}, k_{12}$, and $k_{13}$ versus current for an external field of $3 \mathrm{mT}$.

\section{Solution of Thiele equations}

An effect of spin-torque is generation of magnetization oscillations balancing the Gilbert damping in the free layer. In the case of the vortex only model, the vortex orbit has a frequency that is linearly dependent on the nanocontact current and the vortex orbit radius only depends on the nanocontact parameters independent of the current. To investigate the effect of an antivortex on vortex dynamics in this system, the set of four equations given by Eq. (3) for the vortex and antivortex positions is written in component form,

$$
\begin{gathered}
G R_{V} \dot{\phi}_{V}+\frac{\partial W}{\partial R_{V}}=-\alpha D \dot{R}_{V}, \\
G \dot{R}_{V}+\frac{1}{R_{V}} \frac{\partial W}{\partial \phi_{V}}=-\alpha D R_{V} \dot{\phi}_{V}+G \sigma I \frac{r_{0}^{2}}{R_{V}}, \\
G R_{A} \dot{\phi}_{A}+\frac{\partial W}{\partial R_{A}}=-\alpha D \dot{R}_{A}, \\
G \dot{R}_{A}+\frac{1}{R_{A}} \frac{\partial W}{\partial \phi_{A}}=-\alpha D R_{A} \dot{\phi}_{A} .
\end{gathered}
$$

The simple expression for the spin-torque force in Eq. (12) is the direct result of substitution of Eq. (6) into Eq. (15) and evaluation of the integral. These equations are solved numerically to obtain the vortex and antivortex orbital frequencies as well as orbit radii. It is remarked that the spin-torque term only occurs in the equations describing the vortex motion. This is because there is a large increase in the Oersted energy as the antivortex moves off of the $y$-axis and the spin-torque force will be balanced by the Oersted force if the antivortex center moves only a degree or two off the equilibrium position on the axis. Thus, the force on the antivortex from the VA interaction is the dominant force driving the antivortex. Finally, it is noticed that Eqs. (11), (12) with the linear potential for 
the vortex simply result in a linear dependence of frequency on current reported in Ref. 21.

The parameters used in the numerical solution of Eqs. (11)-(14) are $2 M_{s} \gamma=30 \mathrm{GHz}, \quad M_{s}=8 \cdot 10^{5} \mathrm{~A} / \mathrm{m}$, $\alpha=0.01, r_{0}=50 \mathrm{~nm}$ with the combination of $L=5 \mathrm{~nm}$ and $P=0.3$ is used as an adjustable parameter that results in a vortex orbit radius of about $100 \mathrm{~nm}$. For a small value of the external field ( $B=1 \mathrm{mT}$ ) solution of the Thiele equations shows that both the vortex and antivortex frequencies are equal, but with a phase difference of about 180 degrees with the vortex leading the antivortex by a few degrees because of dissipation. This is the result expected for the antivortex in a quadratic potential driven by the vortex orbital motion and locked into the driving frequency of the vortex. The frequency versus current is illustrated in Fig. 8, which is clearly linear at higher current values with a slope of $50-60 \mathrm{MHz} / \mathrm{mA}$ as predicted by the vortex only models. When the external field is increased to $3 \mathrm{mT}$ the value of $Y_{0}$ decreases, thereby increasing the VA interaction, the effect of which is also shown in Fig. 8. At lower values of the current the current-dependent frequency is definitely nonlinear with a slope of about $26 \mathrm{MHz} / \mathrm{mA}$ for low values of the current.

Next consider how the vortex and antivortex radii are affected by the antivortex bearing in mind that an increasing field or decreasing current will move the antivortex closer to the nanocontact center increasing the vortexantivortex interaction. The vortex orbit is almost circular and the typical antivortex orbit exhibits a larger eccentricity than the vortex orbit. For an external field of $3 \mathrm{mT}$ the vortex orbit radii and the mean antivortex radii are compared in Fig. 9. Here notice the small change in the vortex orbit radius as the current is decreased to $4 \mathrm{~mA}$ with a corresponding increase in the antivortex mean orbit radius as expected since the VA interaction increases with decreasing current. For comparison, the mean antivortex orbit radii for a field of $1 \mathrm{mT}$ are also in Fig. 9 showing the de-

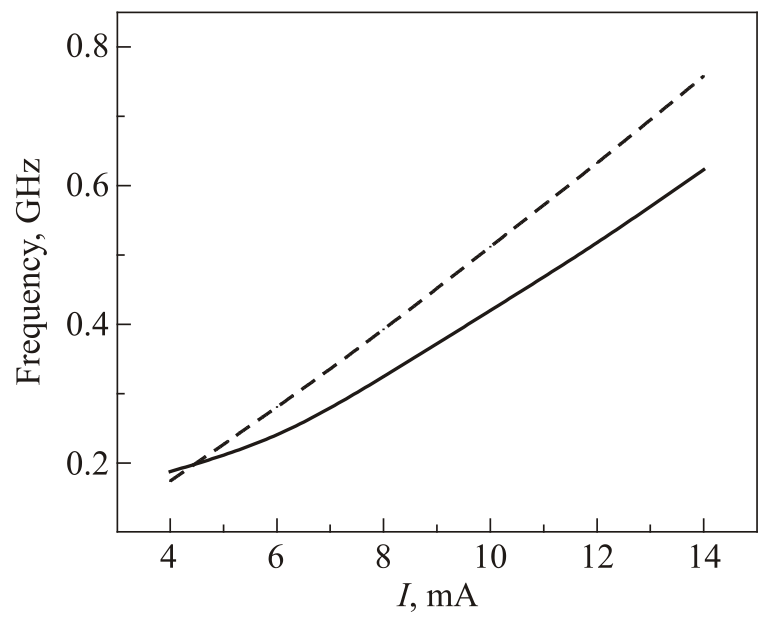

Fig. 8. Frequency versus current for external fields of $1 \mathrm{mT}$ (dashed) and $3 \mathrm{mT}$ (solid).

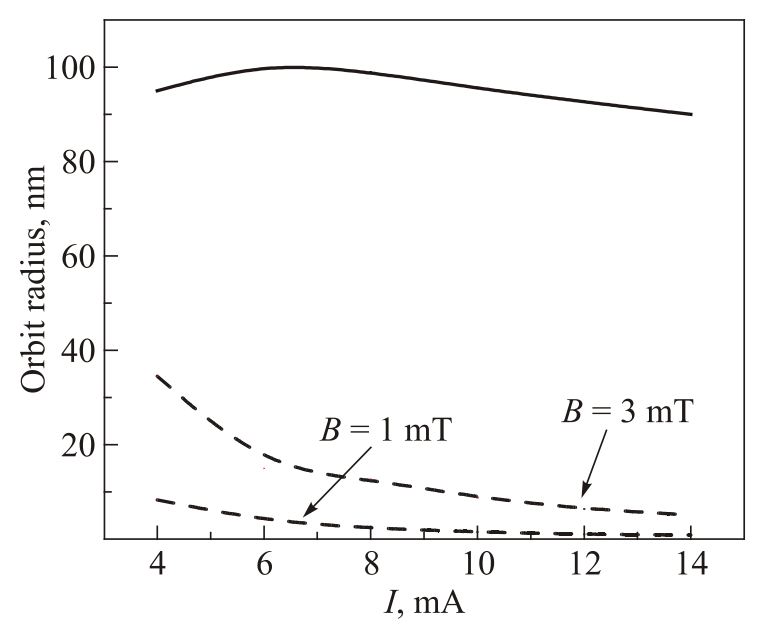

Fig. 9. Mean orbit radii versus current. Solid curves correspond to the vortex orbit and dashed curves correspond to antivortex orbits.

pendence of the antivortex radii on external field. Using these orbit radii it is possible to understand why the sub$\mathrm{GHz}$ mode was not observed [20] for in-plane fields larger than $4 \mathrm{mT}$ when the $\pi$ phase shift between the vortex and antivortex motion is also taken into consideration. For small values of the field the sum of the vortex and antivortex radii will always be much less than the static VA separation so the VA pair will always be wellseparated. However, for an external field of $3 \mathrm{mT}$ and nanocontact current of $4 \mathrm{~mA}$ with a VA separation of $Y_{0}=267 \mathrm{~nm}$, solution of the Thiele equations gives mean vortex and antivortex orbit radii of $95 \mathrm{~nm}$ and $35 \mathrm{~nm}$, respectively so the phase shift will give a closest VA distance of about $140 \mathrm{~nm}$. Therefore, for smaller currents and larger fields the separation will be further decreased and the VA attracting forces such as exchange and the dipolar forces between cores can result in VA annihilation. This process is beyond the scope of the current work, but it is obvious that larger fields and smaller currents will allow small VA separations.

\section{Further considerations and conclusion}

Hysteresis effects can be considered by looking at the energy required to form a vortex-antivortex pair in the form of a Belavin-Polyakov soliton, which is $E_{B P}=4 \pi A$ independent of the VA separation, where $A=1.3 \cdot 10^{-11} \mathrm{~J} / \mathrm{m}$ is the inhomogeneous exchange constant. The critical current for formation is defined to be where the increase, $E_{B P}$ is balanced by the decrease in energy from the Oersted field, which for small $Y_{0} / r_{0}$ is

$$
W_{O e} \approx-\mu_{0} M_{s} I /\left[Y_{0}+2 Y_{0} \log \left(R / r_{0}\right)\right] .
$$

For VA formation it is assumed that the cores are separated by more than $2 l_{0}$ so $Y_{0}$ is chosen to be in the range 10-20 $\mathrm{nm}$, and for typical values of the upper integration limit, $R$ 
the critical current for VA formation is $10-15 \mathrm{~mA}$. After formation of the pair spin-torque drives the cores out of the nanocontact to a stable orbit radius where the pair remains stable to lower values of the current. However, for larger values of the in-plane field the value of $Y_{0}$ decreases until the vortex and antivortex orbits become close and annihilation of the pair occurs. This was observed at a field of $4 \mathrm{mT}$ in [20] and at this field with $I=12 \mathrm{~mA}$ it is noticed that the orbits will overlap qualitatively explaining the origin of the hysteresis effects.

In summary, numerical solution of the Thiele equations for both the vortex and antivortex indicate that effects can observed in nanocontact systems that result from the interaction of the vortex and antivortex. For example, a GMR devise can only detect time-dependent changes in the magnetization at the nanocontact, which is mainly influenced by orbital motion of the vortex leading to the linear dependence of the frequency on current for large VA separations. However, as the external field is increased, the increasing VA interaction allows the development of nonlinearity in the frequency versus current.

\section{Appendix A}

Expansion of $m_{\chi}$ and $\cos \phi$ in series of $R_{V}$ and $R_{A}$ results in the coefficients of Eqs. (8) and (9),

$$
\begin{aligned}
& a_{0}=\frac{Y_{0}-r \sin \chi}{r_{-}}, \\
& b_{0}=\frac{r-Y_{0} \sin \chi}{r_{-}},
\end{aligned}
$$

$a_{22}=\frac{1}{8 r_{-}^{5}}\left[2 r^{2} Y_{0} \cos 2 \chi+2\left(r^{3}+3 r Y_{0}^{2}\right) \sin \chi-4 r^{2} Y_{0}-2 Y_{0}^{3}\right]$

$$
b_{22}=\frac{1}{8 r_{-}^{5}}\left[2 r^{2} Y_{0} \cos 2 \chi+\left(r^{3}+3 r Y_{0}^{2}\right) \sin \chi-2 r^{2} Y_{0}-Y_{0}^{3}\right] \text {, }
$$

$$
\begin{gathered}
a_{12}=\frac{1}{8 r r_{-}^{3}}\left(Y_{0}-r \sin \chi\right)^{2} \sin \phi_{V} \sin \phi_{A}, \\
a_{13}=\frac{1}{8 r r_{-}^{3}}\left(Y_{0}-r \sin \chi\right)^{2} \sin \chi \cos \phi_{V} \cos \phi_{A}, \\
b_{12}=\frac{\cos ^{2} \chi}{8 r_{-}^{2}}\left(r-Y_{0} \sin \chi\right) \sin \phi_{V} \sin \phi_{A}, \\
b_{13}=\frac{1}{8 r r_{-}^{3}}\left[\left(r^{2}+3 Y_{0}^{2}\right) \sin \chi-r Y_{0}\left(1+\sin ^{2} \chi\right)\right] \times \\
\quad \times \sin \chi \cos \phi_{V} \cos \phi_{A},
\end{gathered}
$$

where $r_{-}=\sqrt{r^{2}+Y_{0}^{2}-2 r Y_{0} \sin \chi}$.
The first term in the Eq. (10) is calculated by numerical integration of

$k_{1}=I \int_{0}^{r_{0}} \int_{0}^{2 \pi} m_{\chi} \frac{r^{2}}{r_{0}^{2}} d r d \chi+I \int_{r_{0}}^{R} \int_{0}^{2 \pi} m_{\chi} d r d \chi+\frac{2 \pi}{\mu_{0}} B \int_{0}^{R} \int_{0}^{2 \pi} \cos \phi r d r d \chi$,

using $m_{\chi}=\sin \phi \cos \chi-\cos \phi \sin \chi$ and the ansatz (6) for the magnetization azimuthal angle, $\phi$. The remaining coefficients are calculated by numerical integration of

$$
k_{i j}=I \int_{0}^{r_{0}} \int_{0}^{2 \pi} a_{i j} \frac{r^{2}}{r_{0}^{2}} d r d \chi+I \int_{r_{0}}^{R} \int_{0}^{2 \pi} a_{i j} d r d \chi+\frac{2 \pi}{\mu_{0}} B \int_{0}^{R} \int_{0}^{2 \pi} b_{i j} r d r d \chi
$$

\section{Appendix B}

The correction to the angle $\phi$ from the external magnetic field is found from a variation of both the exchange and Zeeman energy resulting in the nonlinear partial differential equation

$$
l_{0}^{2} \nabla^{2} \phi-h \sin \phi=0
$$

where $h=B / \mu_{0} M_{s}, l_{0}^{2}=2 A / \mu_{0} M_{s}$ is the exchange length $\left(A=1.3 \cdot 10^{-11} \mathrm{~N}\right.$ and $\left.l_{0}=5.7 \mathrm{~nm}\right)$. This equation is linearized by $\phi=\phi_{0}+y(r) \sin \chi$ with $\nabla^{2} \phi_{0}=0$ and $\phi_{0}$ represents the ansatz of Eq. (6). The right hand side is linearized by expansion of $b_{0}$ for small $Y_{0} / r$ results in the nonhomogeneous linear equation,

$$
z^{2} y^{\prime \prime}(z)+z y^{\prime}(z)-\left(1+z^{2}\right) y(z)=\sqrt{h} \frac{Y_{0}}{l_{0}} z,
$$

with the variable change, $z=\sqrt{h}\left(r / l_{0}\right)$.

This equation is solved using variation of parameters subject to the conditions, $y\left(z_{0}\right)=\varepsilon$ and $y\left(z_{m}\right)=0$ where $z_{0}=\sqrt{h}\left(Y_{0+} / l_{0}\right)$ and $z_{m}=\sqrt{h}\left(R_{m} / l_{0}\right)$. Here $Y_{0+}$ is slightly larger than the equilibrium value and $z_{m} \approx 50$ which is well outside of the VA region. The general solution is

$$
y(z)=c_{1} K_{1}(z)+c_{2} I_{1}(z)+M\left(z_{0}, z\right),
$$

where $K_{1}(z)$ and $I_{1}(z)$ are modified Bessel functions and the remaining functions are,

$$
\begin{gathered}
M\left(z_{0}, z\right)=\sqrt{h} \frac{Y_{0}}{l_{0}} \int_{z_{0}}^{z}\left[K_{1}(t) I_{1}(z)-I_{1}(t) K_{1}(z)\right] d t, \\
c_{1}=\frac{\varepsilon I_{1}\left(z_{m}\right)+M\left(z_{0}, z_{m}\right) I_{1}\left(z_{0}\right)}{K_{1}\left(z_{0}\right) I_{1}\left(z_{m}\right)-K_{1}\left(z_{m}\right) I_{1}\left(z_{0}\right)} \\
c_{2}=-\frac{\varepsilon K_{1}\left(z_{m}\right)+M\left(z_{0}, z_{m}\right) K_{1}\left(z_{0}\right)}{K_{1}\left(z_{0}\right) I_{1}\left(z_{m}\right)-K_{1}\left(z_{m}\right) I_{1}\left(z_{0}\right)}
\end{gathered}
$$

For small values of $Y_{0} / r$, the deviation of the ansatz from the $x$-axis is $\Delta \phi=Y_{0} / r$ and the correction from the 
external field is $\phi^{\prime}=y(r)$. Therefore, the magnetization will be aligned with the $x$-axis when

$$
y(R)=\frac{Y_{0}}{R},
$$

which is solved numerically to obtain as estimate of the integration upper limit, $R$.

1. L.D. Landau and E.M. Lifshitz, Phys. Z. Sow. 8, 153 (1935); see also: L.D. Landau and E.M. Lifshitz, Course of Theoretical Physics, Vol. 8: Electrodynamics of Continuous Media, Nauka, Moscow (1992); Butterworth-Heinemann, Oxford (1993).

2. A.A. Thiele, Phys. Rev. Lett. 30, 230 (1973); A.A. Thiele, J. Appl. Phys. 45, 377 (1974).

3. K. Yu. Guslienko, B.A. Ivanov, V. Novosad, Y. Otani, H. Shima, and K. Fukamichi, J. Appl. Phys. 91, 8037 (2002).

4. K. Yu. Guslienko, W. Scholz, R.W. Chantrell, and V. Novosad, Phys. Rev. B 71, 144407 (2005).

5. B.A. Ivanov and V.A. Stephanovich, Phys. Lett. A 141, 89 (1989).

6. J.P. Park and P.A. Crowell, Phys. Rev. Lett. 95, 167201 (2005).

7. S.-B. Choe, Y. Acremann, A. Scholl, A. Bauer, A. Doran, J. Stöhr, and H.A. Padmore, Science 304, 420 (2004).

8. J. Shibata and Y. Otani, Phys. Rev. B 70, 012404 (2004).

9. A.A. Awad, G.R. Aranda, D. Dieleman, K.Y. Guslienko, G.N. Kakazei, B.A. Ivanov, and F.G. Aliev, Appl. Phys. Lett. 97, 132501 (2010).

10. S.S. Cherepov, B.C. Koop, A.Y. Galkin, R.S. Khymyn, B.A. Ivanov, D.C. Worledge, and V. Korenivski, Phys. Rev. Lett. 109, 097204 (2012).

11. G.M. Wysin, Fiz. Nizk. Temp. 41, 1009 (2015) [Low Temp. Phys. 41, No. 10 (2015)].

12. W.H. Rippard, M.R. Pufall, S. Kaka, S.E. Russek, and T.J. Silva, Phys. Rev. Lett. 92, 027201 (2004).

13. W.H. Rippard, M.R. Pufall, S. Kaka, T.J. Silva, S.E. Russek, and J.A. Katine, Phys. Rev. Lett. 95, 067203 (2005).
14. S. Kaka, M.R. Pufall, W.H. Rippard, T.J. Silva, S.E. Russek, and J.A. Katine, Nature (London) 437, 389 (2005).

15. J.C. Slonczewski, J. Magn. Magn. Mater. 159, L1 (1996).

16. L. Berger, Phys. Rev. B 54, 9353 (1996).

17. S. Kasai, Y. Nakatani, K. Kobayashi, H. Kohno, and T. Ono, Phys. Rev. Lett. 97, 107204 (2006).

18. V.S. Pribiag, I.N. Krivorotov, G.D. Fuchs, P.M. Braganca, O. Ozatay, J.C. Sankey, D.C. Ralph, and R.A. Buhrman, Nature Phys. 3, 498 (2007).

19. X.W. Yu, V.S. Pribiag, Y. Acremann, A.A. Tulapurkar, T. Tyliszczak, K.W. Chou, B. Bräuer, Z.-P. Li, O.J. Lee, P.G. Gowtham, D.C. Ralph, R.A. Buhrman, and J. Stöhr, Phys. Rev. Lett. 106, 167202 (2011).

20. M.R. Pufall, W.H. Rippard, M.L. Schneider, and S.E. Russek, Phys. Rev. B 75, 140404 (2007).

21. Q. Mistral, M. van Kampen, G. Hrkac, J.-V. Kim, T. Devolder, P. Crozat, C. Chappert, L. Lagae, and T. Schrefl, Phys. Rev. Lett. 100, 257201 (2008).

22. T. Devolder, J.-V. Kim, P. Crozat, C. Chappert, M. Manfrini, M. van Kampen, W.V. Roy, L. Lagae, G. Hrkac, and T. Schrefl, Appl. Phys. Lett. 95, 012507 (2009).

23. A. Ruotolo, V. Cros, B. Georges, A. Dussaux, J. Grollier, C. Deranlot, R. Guillemet, K. Bouzehouane, S. Fusil, and A. Fert, Nature Nanotech. 4, 528 (2009).

24. T. Devolder, J.-V. Kim, M. Manfrini, W. Van Roy, L. Lagae, and C. Chappert, Appl. Phys. Lett. 97, 072512 (2010).

25. M. Manfrini, T. Devolder, J.-V. Kim, P. Crozat, N. Zerounian, C. Chappert, W. Van Roy, L. Lagae, G. Hrkac, and T. Schrefl, Appl. Phys. Lett. 95, 192507 (2009).

26. F.B. Mancoff, N.D. Rizzo, B.N. Engel, and S. Tehrani, Nature 437, 393 (2005)

27. S.I. Kiselev, J.C. Sankey, I.N. Krivorotov, N.C. Emley, R.J. Schoelkopf, R.A. Buhrman, and D.C. Ralph, Nature 425, 380 (2003).

28. A. Ruotolo, V. Cros, B. Georges, A. Dussaux, J. Grollier, C. Deranlot, R. Guillemet, K. Bouzehouane, S. Fusil, and A. Fert, Nature Nanotech. 4, 528 (2009).

29. C.E. Zaspel, Appl. Phys. Lett. 102, 052403 (2013).

30. A.A. Belavin and A.M. Polyakov, JETP Lett. 22, 245 (1985). 